\title{
The pla gene, encoding plasminogen activator, is not specific to Yersinia pestis
}

\author{
Stephanie Hänsch', Elisabetta Cilli², Giulio Catalano², Giorgio Gruppioni², Raffaella Bianucci ${ }^{1}$, Nils C. Stenseth', \\ Barbara Bramanti ${ }^{1}$ and Mark J. Pallen ${ }^{3^{*}}$
}

\begin{abstract}
Here we present evidence to show that the pla gene, previously thought to be specific to Yersinia pestis, occurs in some strains of Citrobacter koseri and Escherichia coli. This means that detection of this gene on its own can no longer be taken as evidence of detection of Y. pestis.
\end{abstract}

\section{Correspondence}

Molecular assays aimed at detecting traces of the etiological agent of plague, Yersinia pestis, have focused primarily-and sometimes solely-on the plasminogen activator/coagulase (pla) gene [1,2]. This gene is located on the pPCP1 plasmid and has been considered the target of choice for plague detection due to its assumed specificity to $Y$. pestis and its occurrence in multiple copies [3-5]. However, a recent paper about the amplification of the pla gene from tissues from European rats has raised doubts over whether this gene is indeed specific to $Y$. pestis [6]. We can now confirm this suspicion.

We screened archaeological samples from Italy (6th, 14th and 17th centuries CE), amplifying a 70-basepair fragment from the pla gene. Full protocols are described in a previous publication [7], but in brief we performed the work in a dedicated clean laboratory, with physically separated areas for extraction and amplification, following the most stringent criteria for ancient DNA analysis, such as the use of mock extractions and PCR blanks. We used the previously described pla primer pair (Forward primer: GACTGGGTTCGGGCACAT GC-Reverse primer: CGGATGTCTTCTCACGGA). Cycling conditions started with an initial activation step at $95{ }^{\circ} \mathrm{C}$ for $15 \mathrm{~min}$. This was followed by 50 cycles at $94{ }^{\circ} \mathrm{C}$ for $30 \mathrm{~s}$, an assay specific annealing temperature at

\footnotetext{
*Correspondence: m.pallen@warwick.ac.uk

${ }^{3}$ Microbiology and Infection Unit, Warwick Medical School, University of Warwick, Coventry CV4 7AL, UK

Full list of author information is available at the end of the article
}

$60{ }^{\circ} \mathrm{C}$ for $30 \mathrm{~s}$, and $72{ }^{\circ} \mathrm{C}$ for $1 \mathrm{~min}$, ending with a final elongation step at $72{ }^{\circ} \mathrm{C}$ for $10 \mathrm{~min}$. Final cooling was carried out at $8{ }^{\circ} \mathrm{C}$ until analysis.

Target-fragment amplifications were observed in 17 out of 40 samples. We sequenced one of the fragments and performed a BLASTN search of the NCBI database, which, as expected, revealed full-length identity (70/70 at nucleotide level) with numerous sequences from $Y$. pestis. However, we also found full-length identity with two sequences from outside $Y$. pestis. The first was an annotated contig from a genome assembly of Citrobacter koseri (submitted to GenBank in June 2014, with accession number LK931337). This bacterial species has been recognized as a commensal and pathogen in humans and animals $[8,9]$. The second was from a contig from an unannotated genome assembly of Escherichia coli strain FHI29 (submitted to GenBank in June 2014, with accession number LM995843). This sequence is derived from a human fecal isolate from a case of gastroenteritis in Norway. To conduct a more extensive survey, we then performed BLAST searches with the entire pla gene from $Y$. pestis CO92, which confirmed the high level of similarity $(>98 \%)$ with sequences from the genome assemblies of C. koseri (927/939 identities at nucleotide level) and of the E. coli strain FHI29 (925/939 identities) (Fig. 1). The relevant contig from $C$. koseri contained sequences annotated with plasmid-related functions, suggesting that the pla gene in this context is also plasmid-encoded.

The presence of pla sequences from outside $Y$. pestis, each derived from a distinct geographical or taxonomic 
Yersinia pestis

Escherichia coli FHI29

Citrobacter koseri

Yersinia pestis

Escherichia coli FHI29

Citrobacter koseri

Yersinia pestis

Escherichia coli FHI29

Citrobacter koseri

Yersinia pestis

Escherichia coli FHI29

Citrobacter koseri

Yersinia pestis

Escherichia coli FHI29

Citrobacter koseri

Yersinia pestis

Escherichia coli FHI29

Citrobacter koseri

Yersinia pestis

Escherichia coli FHI29

Citrobacter koseri

Yersinia pestis

Escherichia coli FHI29

Citrobacter koseri

Yersinia pestis

Escherichia coli FHI29

Citrobacter koseri

Yersinia pestis

Escherichia coli FHI29

Citrobacter koseri

Yersinia pestis

Escherichia coli FHI2

Citrobacter koseri

Yersinia pestis

Escherichia coli FHI29

Citrobacter koseri

Yersinia pestis

Escherichia coli FHI29 721

Citrobacter koseri

Yersinia pestis

Escherichia coli FHI29

Citrobacter koseri

Yersinia pestis

Escherichia coli FHI29 841

Citrobacter koseri

Yersinia pestis

Escherichia coli FHI29 90

Citrobacter koseri
1 ATGAAGAAAAGTTCTATTGTGGCAACCATTATAACTATTCTGTCCGGGAGTGCTAATGCA

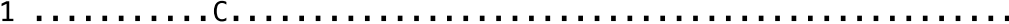

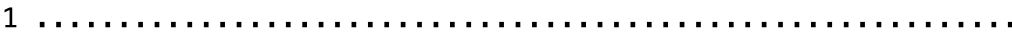

61 GCATCATCTCAGTTAATACCAAATATATCCCCTGACAGCTTTACAGTTGCAGCCTCCACC

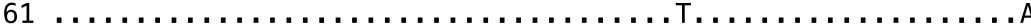

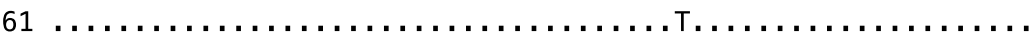

121 GGGATGCTGAGTGGAAAGTCTCATGAAATGCTTTATGACGCAGAAACAGGAAGAAAGATC

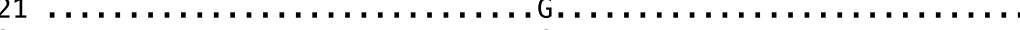

181 AGCCAGTTAGACTGGAAGATCAAAAATGTCGCTATCCTGAAAGGTGATATATCCTGGGAT

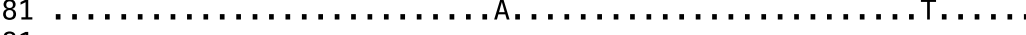

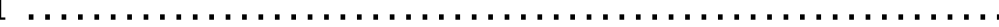

241 CCATACTCATTTCTGACCCTGAATGCCAGGGGGTGGACGTCTCTGGCTTCCGGGTCAGGT

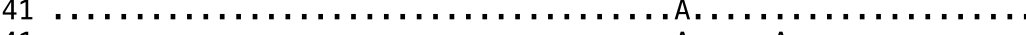

301 AATATGGATGACTACGACTGGATGAATGAAAATCAATCTGAGTGGACAGATCACTCATCT

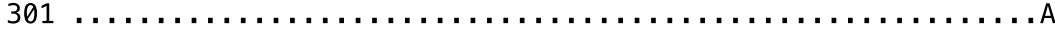

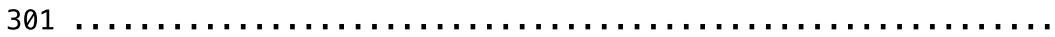

361 CATCCTGCTACAAATGTTAATCATGCCAATGAATATGACCTCAATGTGAAAGGCTGGTTA

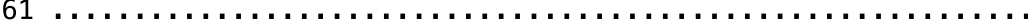

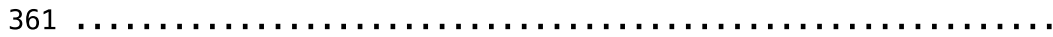

421 CTCCAGGATGAGAATTATAAAGCAGGTATAACAGCAGGATATCAGGAAACACGTTTCAGT

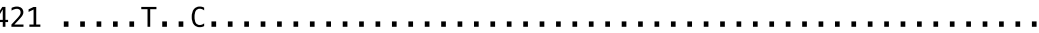

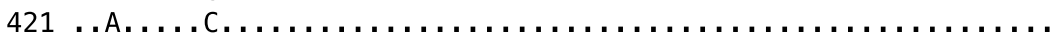

481 TGGACAGCTACAGGTGGTTCATATAGTTATAATAATGGAGCTTATACCGGAAACTTCCCG

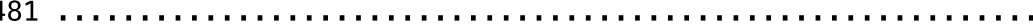

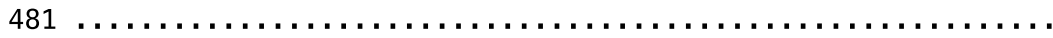

541 AAAGGAGTGCGgGTAATAGgTTATAACCAGCGCTTTTCTATGCCATATATTGGACTTGCA

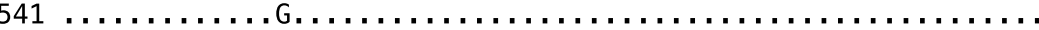

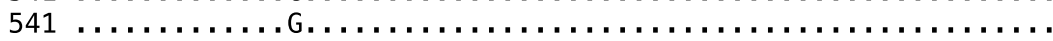

601 GGCCAGTATCGCATTAATGATTTTGAGTTAAATGCATTATTTAAATTCAGCGACTGGGTT

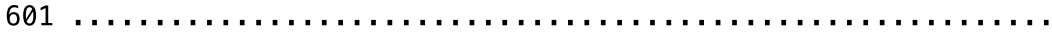

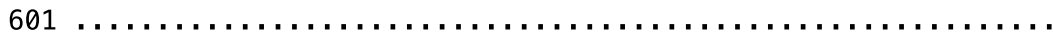

661 CGGGCACATGATAATGATGAGCACTATATGAGAGATCTTACTTTCCGTGAGAAGACATCC

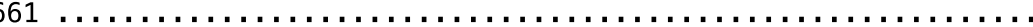

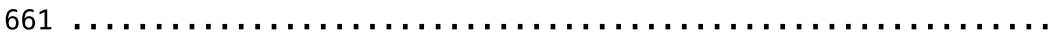

721 GGCTCACGTTATTATGGTACCGTAATTAACGCTGGATATTATGTCACACCTAATGCCAAA



781 GTCTTTGCGGAATTTACATACAGTAAATATGATGAGGGCAAAGGAGGTACTCAGACCATT

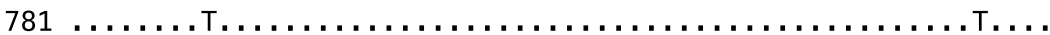

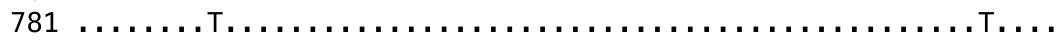

841 GATAAGAATAGTGGAGATTCTGTCTCTATTGGCGGAGATGCTGCCGGTATTTCCAATAAA

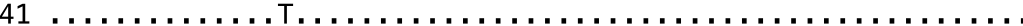

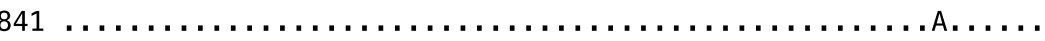

901 AATTATACTGTGACGGCGGGTCTGCAATATCGCTTCTGA

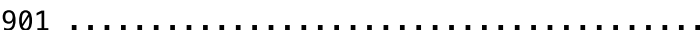

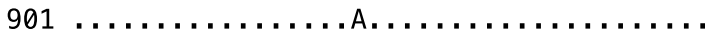

Fig. 1 Multiple alignment of pla sequences from Yersinia pestis and two other species 
setting, confirms beyond doubt that this gene can no longer be considered specific to $Y$. pestis. Although there appear to be some potentially informative sequence differences between the pla sequences from $Y$. pestis and those from other taxa, these findings call into question any results-whether in contemporary diagnostic microbiology or in an ancient DNA setting-that rely on detection of PCR products from this gene alone. Instead, as many researchers in the field already recognise, it is important to obtain sequences from PCR products and detection or identification of $Y$. pestis should rely on sequences from at least two independent molecular targets. More generally, our observations call into question the wisdom of relying on genes from mobile elements as species-specific markers, given the likelihood that such sequences are able to move from one taxon to another. Interestingly, the roles of the sequence differences between the pla genes, some of which are non-synonymous, in the function and evolution of the pla gene product remain to be determined.

\section{Authors' contributions}

EC and GC performed laboratory experiments. SH, GC and MJP performed bioinformatics analyses. RB, GG, NCS and BB conceived of the study, and participated in its design and coordination and helped to draft the manuscript. EC, BB and MJP wrote the manuscript. All authors read and approved the final manuscript.

\section{Author details}

${ }^{1}$ Centre for Ecological and Evolutionary Synthesis, University of Oslo, Oslo, Norway. ${ }^{2}$ Department of Cultural Heritage, University of Bologna, Ravenna Campus, Bologna, Italy. ${ }^{3}$ Microbiology and Infection Unit, Warwick Medical School, University of Warwick, Coventry CV4 7AL, UK.

\section{Compliance with ethical guidelines}

\section{Competing interests}

The authors declare that they have no competing interests.

Received: 9 July 2015 Accepted: 24 September 2015

Published online: 05 October 2015

\section{References}

1. Martin-Alonso A, Soto M, Foronda P, Aguilar E, Bonnet G, Pacheco R, et al. Bartonella spp. and Yersinia pestis reservoirs, Cusco, Peru. Emerg Infect Dis. 2014;20(6):1069-70. doi:10.3201/eid2006.131194.

2. Loïez C, Herwegh S, Wallet F, Armand S, Guinet F, Courcol RJ. Detection of Yersinia pestis in sputum by real-time PCR. J Clin Microbiol. 2003;41(10):4873-5.

3. Sodeinde OA, Goguen JD. Genetic analysis of the 9.5-kilobase virulence plasmid of Yersinia pestis. Infect Immun. 1988;56:2743-8.

4. Norkina OV, Kulichenko AN, Gintsburg AL, Tuchkov IV, Popov YuA, Aksenov MU, et al. Development of a diagnostic test for Yersinia pestis by the polymerase chain reaction. J Appl Bacteriol. 1994;76:240-5.

5. Parkhill J, Wren BW, Thomson NR, Titball RW, Holden MT, Prentice MB, et al. Genome sequence of Yersinia pestis, the causative agent of plague. Nature. 2001;413(6855):523-7.

6. Janse I, Hamidjaja RA, Reusken C. Yersinia pestis plasminogen activator gene homolog in rat tissues. Emerg Infect Dis. 2013;19(2):342-4.

7. Harbeck M, Seifert L, Hänsch S, Wagner DM, Birdsell D, Parise KL, et al. Yersinia pestis DNA from skeletal remains from the 6th Century AD reveals insights into Justinianic plague. PLoS Pathog. 2013;9(5):e1003349. doi:10.1371/journal.ppat.1003349.

8. Komine M, Massa A, Moon L, Mullaney T. Citrobacter koseri Septicaemia in a Holstein Calf. J Comp Pathol. 2014;151(4):309-13. doi:10.1016/j. jcpa.2014.07.005.

9. Doran TI. The role of Citrobacter in clinical disease of children: review. Clin Infect Dis. 1999;28:384-94.

\section{Submit your next manuscript to BioMed Central and take full advantage of:}

- Convenient online submission

- Thorough peer review

- No space constraints or color figure charges

- Immediate publication on acceptance

- Inclusion in PubMed, CAS, Scopus and Google Scholar

- Research which is freely available for redistribution

Submit your manuscript at www.biomedcentral.com/submit

C Biomed Central 